\title{
Aberrant Expression Profile of Long Noncoding RNA in Human Sinonasal Squamous Cell Carcinoma by Microarray Analysis
}

\author{
Ling-zhao Meng, ${ }^{1}$ Ju-gao Fang, ${ }^{2}$ Jing-wu Sun, ${ }^{1}$ Fan Yang, ${ }^{1}$ and Yong-xiang Wei ${ }^{1}$ \\ ${ }^{1}$ Department of Otolaryngology-Head and Neck Surgery, Beijing Anzhen Hospital, Capital Medical University, Beijing 100029, China \\ ${ }^{2}$ Department of Otolaryngology-Head and Neck Surgery, Beijing Tongren Hospital, Capital Medical University, Beijing 100730, China
}

Correspondence should be addressed to Ju-gao Fang; fangjugaotongren@163.com and

Yong-xiang Wei; weiyongxianganzhen@163.com

Received 12 September 2016; Accepted 8 November 2016

Academic Editor: Jan Plzak

Copyright (C) 2016 Ling-zhao Meng et al. This is an open access article distributed under the Creative Commons Attribution License, which permits unrestricted use, distribution, and reproduction in any medium, provided the original work is properly cited.

\begin{abstract}
Objectives. This study aimed to identify aberrantly expressed long noncoding RNAs (lncRNAs) profile of sinonasal squamous cell carcinoma (SSCC) and explore their potential functions. Methods. We investigated lncRNA and mRNA expression in SSCC and paired adjacent noncancerous tissues obtained from 6 patients with microarrays. Gene ontology (GO) analysis and pathway analysis were utilized to investigate the gene function. Gene signal-network and lncRNA-mRNA network were depicted. Quantitative real-time polymerase chain reaction (qRT-PCR) was utilized to validate 5 lncRNAs in a second set of paired SSCC and adjacent noncancerous tissues obtained from 22 additional patients. Results. We identified significantly differentially expressed lncRNAs $(n=3146)$ and mRNAs $(n=2208)$ in SSCC relative to noncancerous tissues. The GO annotation indicated that there are some core gene products that may be attributed to the progress of SSCC. The pathway analysis identified many pathways associated with cancer. The results of lncRNA-mRNA network and gene signal-network implied some core lncRNAs/mRNAs might play important roles in SSCC pathogenesis. The results of qRT-PCR showed that all of the $5 \operatorname{lncRNAs}$ were differentially expressed and consistent with the microarray results. Conclusion. Our study is the first screening and analysis of lncRNAs expression profile in SSCC and may offer new insights into pathogenesis of this disease.
\end{abstract}

\section{Introduction}

Head and neck squamous cell carcinoma is the sixth most common malignancy worldwide. Sinonasal squamous cell carcinomas (SSCC) are rare tumor, estimated to account for approximately 3-6\% of all head and neck squamous cell carcinomas. SSCC originate in the respiratory epithelium of the sinonasal cavities [1]. Approximately $60 \%$ of SSCC arise in the maxillary sinus, $20-30 \%$ in the nasal cavity, $10-15 \%$ in the ethmoid sinuses, and $\sim 1 \%$ in the frontal and sphenoid sinuses $[2,3]$. Environmental factors, such as wood dust and textile, may play a critical role in the development of SSCC. As in other head and neck squamous cell carcinomas, smoking is a known risk factor [4]. Males who have greater occupational exposure to carcinogens are affected twice as often as females. It is controversial that chronic inflammatory sinus disease could influence development of SSCC. Human papilloma virus (HPV) types 16 and 18 may be implicated in malignant transformation of inverted papillomas [5]. The low incidence of SSCC combined with their nonspecific symptoms often leads to a critical delay in diagnosis. Treatment for SSCC is usually primarily surgical with adjuvant radiotherapy and sometimes with adjuvant chemotherapy for all except small tumors [1]. Surgical management of SSCC is of great challenge due to its anatomical complexity, especially advanced SSCC involving eye, skull base, or infratemporal fossa. In spite of major advances in the therapy of SSCC, including surgery and chemoradiotherapy methods, the 5-year survival rate is still very low (30-50\%) [1].

Long noncoding RNAs (lncRNAs) are a subset of noncoding RNAs > 200 nucleotides in length and do not encode any protein. Due to the poor evolutionary conservation relative to the protein coding regions of the genome, lncRNAs were once considered as transcriptional noise or junk and have 
TABLE 1: Clinical parameters of 6 SSCC patients that underwent lncRNA expression profiling.

\begin{tabular}{|c|c|c|c|c|c|c|}
\hline $\begin{array}{l}\text { Specimen } \\
\text { number of } \\
\text { SSCC tissues }\end{array}$ & Gender & Age (years) & Exposure factor & TNM stage & $\begin{array}{c}\text { Histologic } \\
\text { differentiation }\end{array}$ & $\begin{array}{l}\text { Specimen number of } \\
\text { noncancerous tissues }\end{array}$ \\
\hline F5 & Male & 81 & Wood dust & T1N0M0 & Well & G2 \\
\hline F9 & Male & 46 & Tobacco smoke & T3N0M0 & Poorly & G10 \\
\hline A3 & Male & 52 & Tobacco smoke & T4N0M0 & Moderately & B2 \\
\hline D6 & Male & 72 & Wood dust & T4N0M0 & Moderately & $\mathrm{F} 1$ \\
\hline $\mathrm{F} 2$ & Male & 52 & Tobacco smoke & T3N0M0 & Moderately & $\mathrm{H} 2$ \\
\hline D2 & Male & 66 & Tobacco smoke & T3N0M0 & Moderately to Poorly & D9 \\
\hline
\end{tabular}

not been well studied historically. Recently, considerable evidence has been accumulating showing that aberrant expression of lncRNAs contributes to the development of human cancers [6-8]. IncRNAs contribute to tumor development through numerous different cellular processes, ranging from transcriptional and posttranscriptional regulation of relevant genes to the control of cell cycle distribution, cell differentiation, and epigenetic modifications. lncRNAs may be involved in cell proliferation, tumor invasion, metastasis, or apoptosis process. IncRNAs are pervasively transcribed and have a critical role in genome regulation $[6,7,9]$. However, to our knowledge, little is known about lncRNAs expression profile in SSCC, and the potential pathways regulating SSCC invasiveness remain poorly understood.

This pilot study aimed to identify aberrantly expressed lncRNAs profile of SSCC and explore their potential functions. This study will help us to understand the tumorigenesis and development of SSCC and provide some new biomarkers that may be critical to the developmental cascade.

\section{Materials and Methods}

2.1. Patients and Tissue Samples. A total of 28 pairs of primary SSCC tissues and their paired adjacent noncancerous sinonasal tissues were surgically obtained from adult patients undergoing treatment at Anzhen Hospital and Tongren Hospital (two tertiary academic centers in Beijing, China) between January 2013 and August 2014. During surgery, fresh tumor tissue and paired noncancerous tissue isolated from at least $2 \mathrm{~cm}$ away from the tumor border (sometimes contralateral normal sinonasal mucosa) were collected in the operating room and processed immediately in liquid nitrogen within 15 minutes and then stored in RNA Fixer Reagent (Bioteke, Beijing, China) at $-80^{\circ} \mathrm{C}$ prior to total RNA extraction. 6 pairs of tissues underwent microarray analysis (Table 1) and the remaining 22 tissues were used in validation studies by quantitative real-time polymerase chain reaction (qRT-PCR). Tobacco smoke was the most common exposure factor, about $66.67 \%(4 / 6)$ in the microarray analysis series and $63.64 \%(14 / 22)$ in the PCR validation series. Wood dust was the second common exposure factor, about $33.33 \%$ $(2 / 6)$ in the microarray analysis series and $18.18 \%(4 / 22)$ in the PCR validation series. Other exposure factors were chronic sinusitis (3/22) and leather dust (1/22). All cases were reviewed by two or more independent pathologists, and none of the patients had been previously treated with radiotherapy or chemotherapy. All tumor staging was determined according to the tumor-node-metastasis (TNM) staging criteria of American Joint Committee on Cancer (AJCC), 2010.

The Ethics Committee in Clinical Research of Capital Medical University approved this study, and written informed consent was provided by all patients.

2.2. Transcript Analysis. RNA extraction was carried out using standard methods (Life Technologies; RNA Easy, Qiagen, Valencia, CA, USA). Total RNA was quantified by the NanoDrop ND-2000 (Thermo Scientific) and RNA integrity was assessed using Agilent Bioanalyzer 2100 (Agilent Technologies).

Microarray profiling was conducted with the Agilent Human lncRNA ( $4 * 180 \mathrm{~K}$, Design ID: 062918) in this experiment and data analysis of the 12 samples has been completed in the laboratory of the KPS Biotechnology Company in Beijing, China. The sample labeling, microarray hybridization, and washing were performed based on the manufacturer's standard protocols. Briefly, total RNA was transcribed to double strand cDNA and then synthesized into cRNA and labeled with Cyanine-3-CTP. The labeled cRNAs were hybridized onto the microarray. After washing, the arrays were scanned with the Agilent Scanner G2505C.

Feature Extraction software (version 10.7.1.1, Agilent Technologies) was used to analyze array images to obtain raw expression data, which was processed using GeneSpring. Briefly, raw data was normalized with the quantile algorithm. Probes which had at least 1 out of 2 conditions having $75 \%$ flags in " $p$ " were selected for further data analysis. Differentially expressed gene transcripts were later identified. We set a standard threshold set for up- and downregulated genes of a fold change $\geq 2.0$ and a $p$ value $\leq 0.05$.

Hierarchical clustering was performed to display expression patterns among samples. Briefly, we calculated the distance matrix between the gene expression data. Once this matrix of distances was computed, clustering begins. Agglomerative hierarchical processing consisted of repeated cycles where the two closest remaining items (those with the smallest distance) are joined by a node/branch of a tree, with the length of the branch set to the distance between the joined items. The two joined items were removed from the list of 
TABLE 2: The primer sequences in the present study.

\begin{tabular}{lccc}
\hline lncRNA name & Forward primers $\left(5^{\prime}-3^{\prime}\right)$ & Reverse primers $\left(5^{\prime}-3^{\prime}\right)$ & Amplicon size $(\mathrm{bp})$ \\
\hline NONHSAT066780 & GGAACCAGCTACTCCACACC & CCTACCCAGGCCAAGTTCTG & 182 \\
TCONS_12_00030809 & TGAAAAACCACAGGCCCACT & ACAAACATGTCTCTCATCAGCAC & 78 \\
NONHSAT125629 & GATTTGAATCGGTCGGCGG & AGGCATTTCCTCTCACGCC & 284 \\
NONHSAG040260 & ATGCCCTACGAATGTGGACC & TCGGCCCACTGCTAAACATC & 218 \\
NONHSAG043195 & GGGAAGGCTGCCTATGAAGG & AATTCGGGGTTGCAGGTTCT & 184 \\
\hline
\end{tabular}

items being processed and replaced by an item that represents the new branch. The distances between this new item and all other remaining items were computed, and the process was repeated until only one item remained.

2.3. IncRNA-mRNA Coexpression Networks. $R$ function cor. test (a test for association/correlation between paired samples) was utilized to compute Pearson's correlation coefficient to measure the gene coexpression. The lncRNAs/mRNAs (Pearson correlation coefficients $\geq 0.93$ ) were selected to draw the network with Cytoscape.

According to these data, we built lncRNA-mRNA network using the correlation coefficients to examine interactions between lncRNA and mRNA. The value of "degree" in coexpression network indicated that one mRNA/lncRNA might be correlated with several lncRNAs/mRNAs.

2.4. GO Analysis and KEGG Pathway Analysis. GO analysis was applied to analyze the main function of the differential expression genes according to the GO database. Pathway analysis was used to find out the significant pathway of the differential genes according to KEGG. We used Fisher's exact test and $\chi^{2}$ tests to select the significant pathway, and the threshold of significance was defined by $p$ value and false discovery rate (FDR). The enrichment Re was calculated using standard methods with a $p$ value (hypergeometric- $p$ value) denoting the significance of the pathway correlated with the conditions, with a threshold of $p<0.05$, adjusted for multiple comparisons.

2.5. Gene Signal-Network. Gene-gene interaction network was constructed based on the data of differentially expressed genes. Java was utilized to build and analyze molecular networks. After parsing the whole KEGG database, selected genes involved in relevant pathways were extracted, and the study pathway network was generated with the help of the pathway topology in the KEGG database.

2.6. qRT-PCR Analysis. Total RNA was extracted and purified using standard methods (Life Technologies; RNA Easy, Qiagen, Valencia, CA, USA). M-MLV reverse transcription (Promega) was utilized to synthesize cDNA. 5 lncRNA expressions in sinonasal tissues were measured by qRT-PCR which was performed on the ABI 7500 qPCR system with the primer pairs listed in Table 2 . The raw quantifications were normalized to the beta-actin gene values for each sample and fold changes were shown as mean \pm SD in three independent experiments, each in triplicate.

2.7. Statistical Analysis. All data were expressed as the mean $\pm \mathrm{SD}$ or proportions where appropriate. Expression levels between SSCC tissues and adjacent nontumor tissues were analyzed by paired-sample $t$-tests. $p$ values $<0.05$ (two-tailed) indicated statistical significance. The Statistical Program for Social Sciences (SPSS) 21.0 software (SPSS, Chicago, IL, United States) was employed to perform all of the statistical analyses.

\section{Results}

3.1. Overview of $\operatorname{lnc} R N A$ Profile. Out of a collection of 78,243 lncRNAs and 32,776 mRNAs probes, our lncRNA expression profile of 6 malignant sinonasal tissue and corresponding normal tissue samples from patients with SSCC indicated dysregulation of $6.73 \%$ (821 upregulated and 1103 downregulated transcripts) of mRNA and $4.02 \%$ (1174 upregulated and 1098 downregulated transcripts) of lncRNA transcripts in SSCC tissues (fold change $>2, p<0.05$ ) (Figure 1). As expected, the lncRNA and mRNA expression profiles allowed distinguishing malignant and normal tissue samples accurately based on the molecular signature.

Out of the group of RNAs that were upregulated, lncRNA NONHSAT096777 and mRNA HORMAD1 showed the greatest degree of demonstrated upregulation, with 212.076and 91.757-fold increases, respectively; of those that were downregulated, IncRNA TCONS_12_00002973 and mRNA ANKRD30A demonstrated the greatest degree of downregulation, with 298.204- and 275.902-fold decreases, respectively (Tables 3 and 4).

Hierarchical clustering of the lncRNAs and mRNAs profile was performed using cluster 3.0.2; hierarchical clustering of the expression of the top 100 dysregulated lncRNAs and top dysregulated 100 mRNAs based on centered Pearson correlation clearly separated SSCC tissues from corresponding normal tissues (Figure 2).

3.2. IncRNA-mRNA Coexpression Network. We constructed the lncRNA-mRNA coexpression network to identify the interactions between mRNAs and lncRNAs. The results showed that the coexpression network was composed of 787 network nodes and 8478 connections between 445 lncRNAs and 342 mRNAs. Within this coexpression network, 7635 


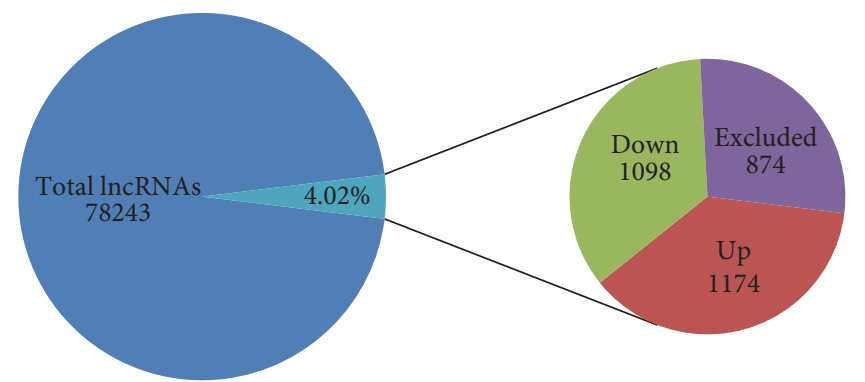

Total lncRNAs: 78243

Significantly different expressed lncRNAs: 3146 (4.02\%)

Upregulated lnRNAs (screened): 1174

- Downregulated lncRNAs (screened): 1098

Excluded lncRNAs: 874

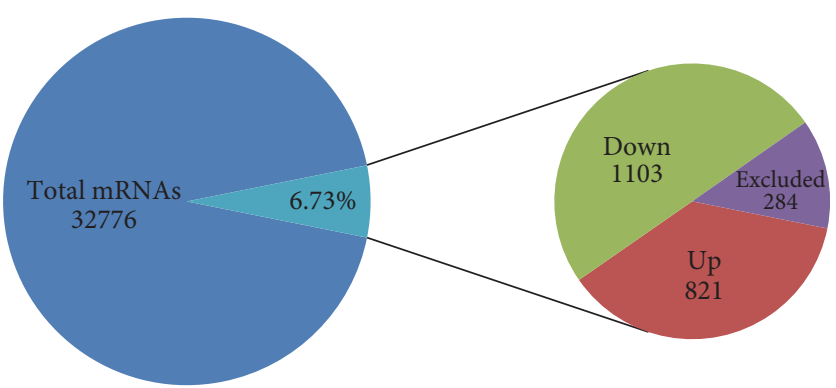

Total mRNAs: 32776

- Significantly different expressed mRNAs: 2208 (6.73\%)

- Upregulated mRNAs (screened): 821

- Downregulated mRNAs (screened): 1103

Excluded mRNAs: 284

(a)

(b)

FIGURE 1: (a) Brief microarray results of lncRNAs. Expression levels of 78,243 lncRNAs were assessed in 6 pairs of SSCC tissues and paired adjacent noncancerous sinonasal tissues using Agilent Human lncRNA $4 * 180 \mathrm{~K}$ microarrays. Compared with paired adjacent noncancerous tissues, 3146 lncRNAs (4.02\%) had significant changes in expression levels (fold change $>2, p<0.05$ ). A total of 874 lncRNAs were excluded due to low expression levels. A total of 3146 lncRNAs were then identified from the screen, with 1174 upregulated and 1098 downregulated. (b) Brief microarray results of mRNAs. Expression levels of 32,776 mRNAs were assessed in 6 pairs of SSCC tissues and paired adjacent noncancerous sinonasal tissues using Agilent Human lncRNA $4 * 180 \mathrm{~K}$ microarrays. Compared with paired adjacent noncancerous tissues, 2208 mRNAs (6.73\%) had significant changes in expression levels (fold change $>2, p<0.05$ ). A total of 284 mRNAs were precluded due to low expression levels. A total of 2208 lncRNAs were then identified from the screen, with 821 upregulated and 1103 downregulated.

TABLE 3: Top 20 aberrantly expressed lncRNAs in microarray for 6 pairs of SSCC and paired adjacent noncancerous sinonasal tissues.

\begin{tabular}{|c|c|c|c|c|c|c|}
\hline Probe name & $p$ & $\mathrm{FC}(\mathrm{abs})$ & Regulation & ncRNA accession & Gene symbol & Chr \\
\hline CUST_13287_PI429545395 & 0.019137 & 239.3843 & Down & NONHSAT096777 & STATH & $\operatorname{chr} 4$ \\
\hline CUST_21727_PI429545388 & 0.039463 & 84.90894 & Down & NONHSAT009094 & PIGR & chrl \\
\hline CUST_76033_PI429545399 & 0.024086 & 82.32558 & Down & NONHSAT013344 & MSMB & $\operatorname{chr} 10$ \\
\hline CUST_5401_PI429545406 & 0.024154 & 78.48107 & Down & NONHSAG018364 & NONHSAG018364 & chr16 \\
\hline CUST_82017_PI429545376 & $9.06 E-04$ & 76.64131 & Down & TCONS_12_00029708 & linc-RUSC2 & chr9 \\
\hline CUST_13257_PI429545395 & 0.019957 & 75.85599 & Down & NONHSAT096773 & STATH & $\operatorname{chr} 4$ \\
\hline CUST_13277_PI429545395 & 0.023234 & 74.90795 & Down & NONHSAT096776 & STATH & chr4 \\
\hline CUST_30951_PI429545395 & $9.49 E-05$ & 67.20975 & Down & NONHSAT100745 & CTD-2351A8.1 & chr5 \\
\hline CUST_91115_PI429545399 & 0.04924 & 65.20921 & Down & NONHSAT017390 & MUC5B & chr11 \\
\hline CUST_5411_PI429545406 & 0.020191 & 56.05385 & Down & NONHSAT147874 & ZG16B & chr16 \\
\hline CUST_17568_PI429545406 & 0.02086 & 53.66862 & Down & NONHSAT143547 & HP & chr16 \\
\hline CUST_32391_PI429545395 & 0.001238 & 51.26765 & Down & NONHSAT101166 & $\mathrm{C} 7$ & chr5 \\
\hline CUST_57295_PI429545380 & $1.32 E-04$ & 49.87698 & Down & FR352905 & FR352905 & chr19 \\
\hline CUST_15837_PI429545395 & $7.74 E-04$ & 47.76275 & Down & NONHSAT097405 & MMRN1 & $\operatorname{chr} 4$ \\
\hline CUST_32401_PI429545395 & 0.001893 & 41.53056 & Down & NONHSAT101168 & C7 & chr5 \\
\hline CUST_58955_PI429545388 & $1.03 E-05$ & 40.74976 & Up & NONHSAT075623 & HOXD11 & $\operatorname{chr} 2$ \\
\hline CUST_72258_PI429545388 & $2.00 E-04$ & 39.85786 & Down & NONHSAT087567 & CHL1 & chr3 \\
\hline CUST_58985_PI429545388 & $5.09 E-06$ & 38.35 & $\mathrm{Up}$ & NONHSAT075626 & HOXD10 & chr2 \\
\hline CUST_32370_PI429545395 & 0.007453 & 33.21905 & Down & NONHSAG040260 & NONHSAG040260 & chr5 \\
\hline CUST_24853_PI429545410 & 0.003046 & 31.34336 & Down & XR_253519.1 & LOC101928556 & N/A \\
\hline
\end{tabular}

FC, fold change; Chr, chromosome; N/A, not annotated.

pairs connections presented as positive, and 843 pairs connections presented as negative (Figure 3). This coexpression network indicated that one lncRNA (NONHSAT041869) could target 118 mRNAs/mRNAs at most and one mRNA (EXO1) could correlate with 122 lncRNAs/mRNAs at most.
The results implied that EXO1, CDCA5, and BUB1B may play key roles in SSCC process and development.

3.3. Function Analysis of Differentially Expressed Genes. Functional roles of lncRNAs can only be indirectly predicted 


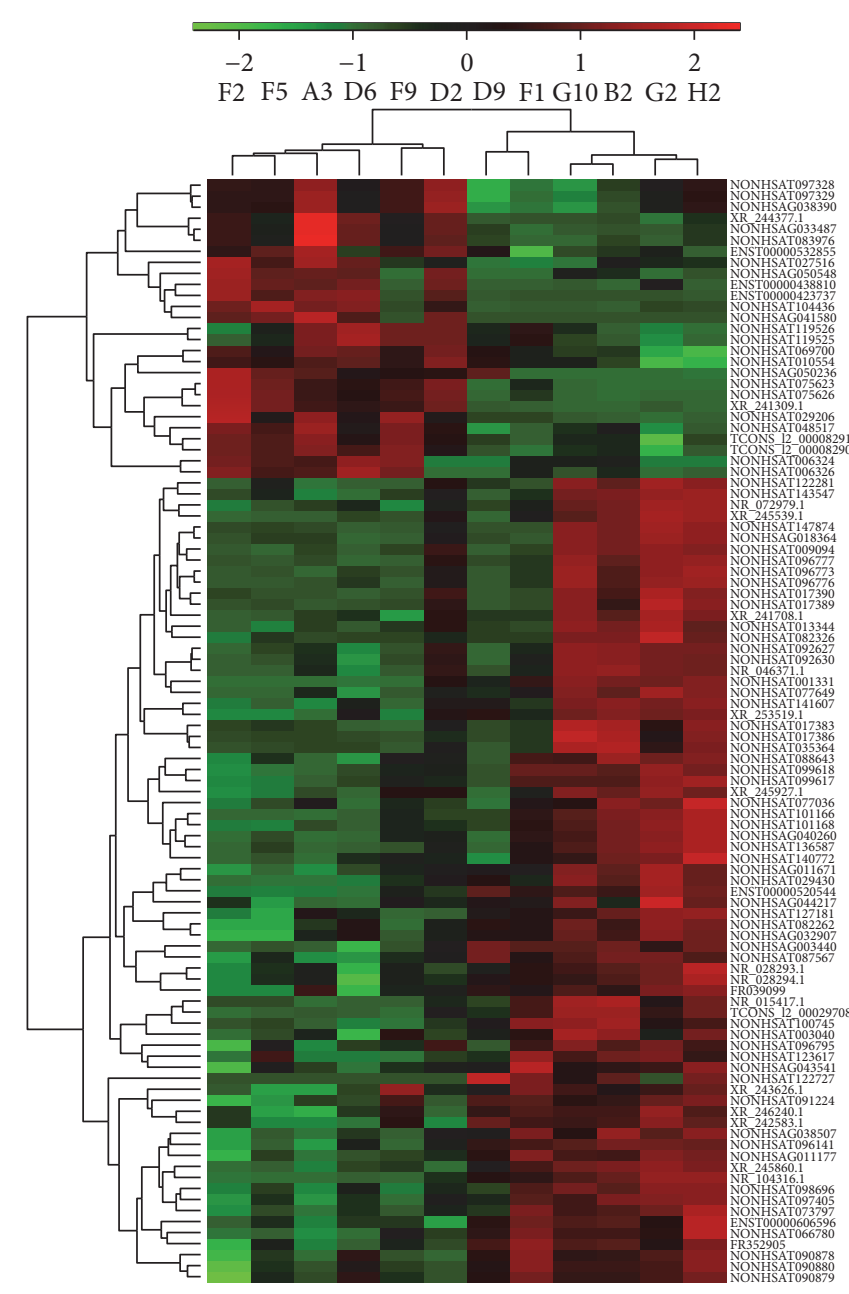

(a)

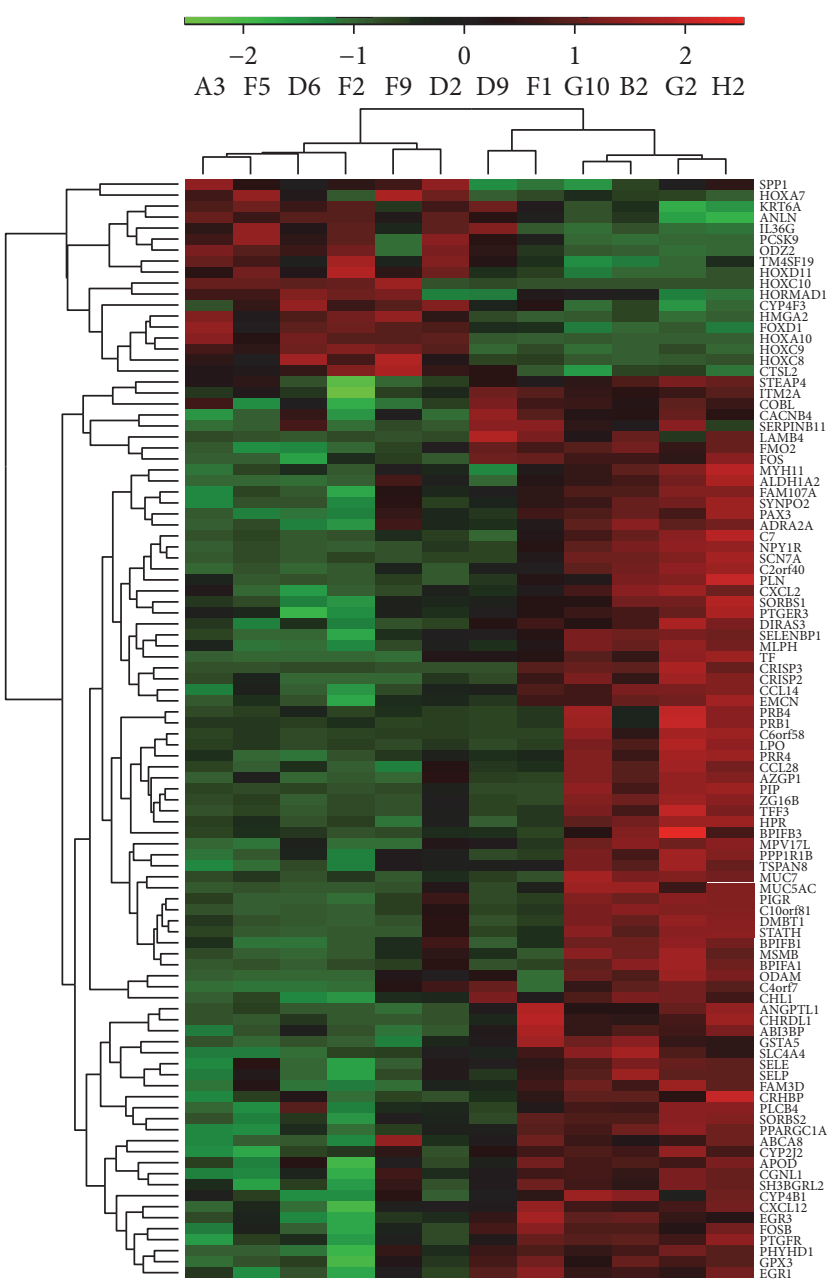

(b)

FIGURE 2: Heat map of lncRNAs and mRNAs that were often aberrantly expressed in SSCC compared with paired adjacent noncancerous sinonasal tissues. (a) Hierarchical clustering analysis of the top 100 dysregulated lncRNAs. (b) Hierarchical clustering analysis of the top 100 dysregulated mRNAs. Each row represents one lncRNA or mRNA, and each column represents one tissue sample. The relative lncRNA or mRNA expression is depicted according to the color scale. Red indicates elevated expression and green indicates reduced expression. 2, 0 and -2 are fold changes in the corresponding spectrum. Carcinoma group (A3, F5, D6, F2, F9, and D2). Paired adjacent noncancerous group (D9, F1, G10, B2, G2, and H2).

by analyzing the functions of their coexpressed mRNAs, because most lncRNAs' functions have not yet been defined. To investigate underlying biological associations, we ran GO and KEGG pathway analysis on the top 500 differentially expressed lncRNAs and mRNAs. GO analysis indicated that these differentially expressed genes were enriched in 12 biological processes; the majority were proven to be related to cancer-associated biological behaviors; the top 3 were multicellular organismal development, mitotic cell cycle, and cell cycle. The differentially expressed genes also were enriched in 12 cellular components; the top 3 were nucleus, extracellular region, and cytosol. Similarly, 12 molecular functions were enriched for including protein binding, DNA binding, and ATP binding. KEGG analysis revealed pathways associated with cancer, such as microRNAs in cancer, p53 signaling pathway, and PI3K-Akt signaling pathway (Figures $4(\mathrm{a})-4(\mathrm{~d}))$.
3.4. Gene Signal-Network. We performed a signal-net analysis to investigate the global network, based on the significantly regulated KEGG. With signal-net, we screened the important dysregulated genes involved in the differences between SSCC and normal tissues (Figure 5). The results showed that the core genes may have played an important role in SSCC process. According to the results of this analysis, the top 3 betweenness genes were MAPK12, RAPGEF3, and KIT.

3.5. qRT-PCR Validation. Five differentially expressed lncRNAs were randomly selected for validation by means of qRT-PCR according to the manufacturer's recommendations. NONHSAT125629 and TCONS_12_00030809 were upregulated and NONHSAT066780, NONHSAG040260, and NONHSAG043195 were downregulated in SSCC. The results of qRT-PCR were consistent with those of the microarray. All 


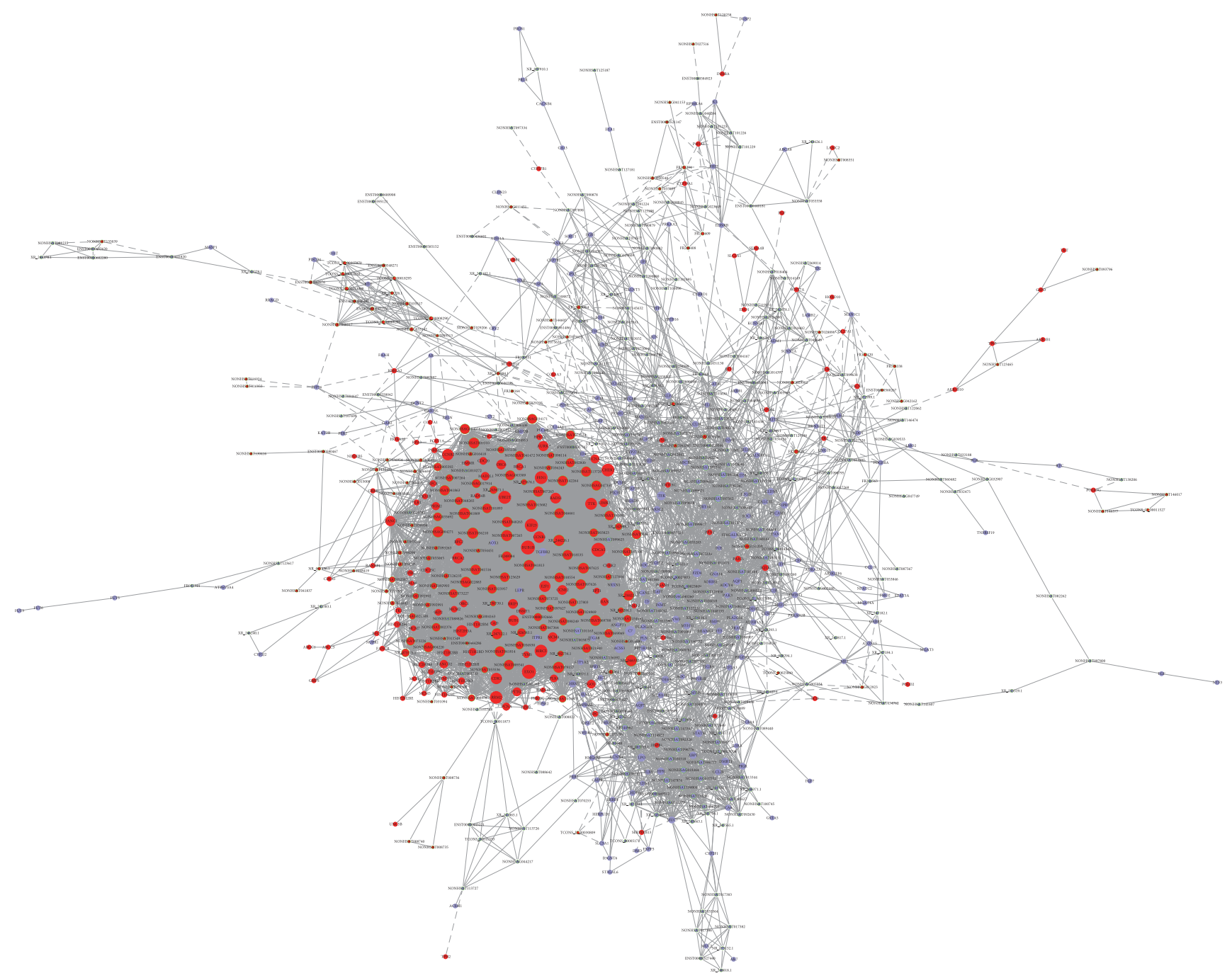

FIGURE 3: IncRNA-mRNA coexpression network. The SSCC consisted of coexpression relationships between lncRNAs and mRNAs. The red circles denote mRNAs and the blue circles denote lncRNAs. The node degree is indicated by the circle size. An edge represents a coexpression relationship between mRNA and a lncRNA in the context of SSCC progression.

of the $5 \mathrm{lncRNAs}$ were differentially expressed with the same trend (up- or downregulated) (Figure 6).

\section{Discussion}

SSCC is a rare disease arising in the epithelium of respiratory tract and is very poorly studied from the molecular perspective. To date, the pathogenesis of SSCC remains unclear due to its low incidence. Only a few studies have focused on its pathogenesis and potential molecular targets for therapy, specifically microRNAs [10-12]. Recently, studies have increasingly shown that many types of tumors are closely associated with the abnormal expression of lncRNAs [6-9]. In head and neck cancer, tongue cancer [13], laryngeal cancer [14], nasopharyngeal cancer [15], and thyroid cancer [6] are all associated with the abnormal expression of lncRNAs. However, to the best of our knowledge, there are no reports on lncRNA expression profiles in SSCC.
Here, we investigated the lncRNA and mRNA expression profiles of SSCC samples from patients using microarray analysis. We identified thousands of lncRNAs that are expressed significantly differently in SSCC compared to adjacent noncancerous tissues, including both upregulation and downregulation. To some extent, false positive results do exist in the microarray detection. Therefore, 5 lncRNAs were randomly selected to validate the microarray results. Consistent with the microarray results, all of the 5 lncRNAs were differentially expressed based on the results of qRTPCR.

In this study, we used GO and KEGG pathway analyses to identify biological functions enriched among the differentially expressed mRNAs. We found that these mRNAs were involved in a lot of cancer-associated biological processes, cellular components, and molecular functions. The GO annotation indicated that these gene products may affect the tumorigenesis and development of SSCC. The 


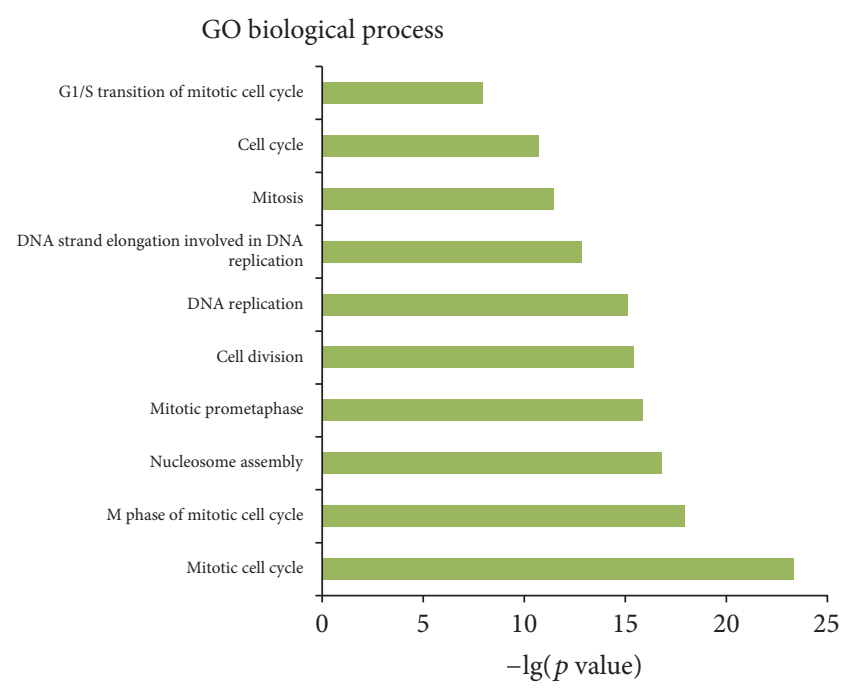

(a)

GO molecular function

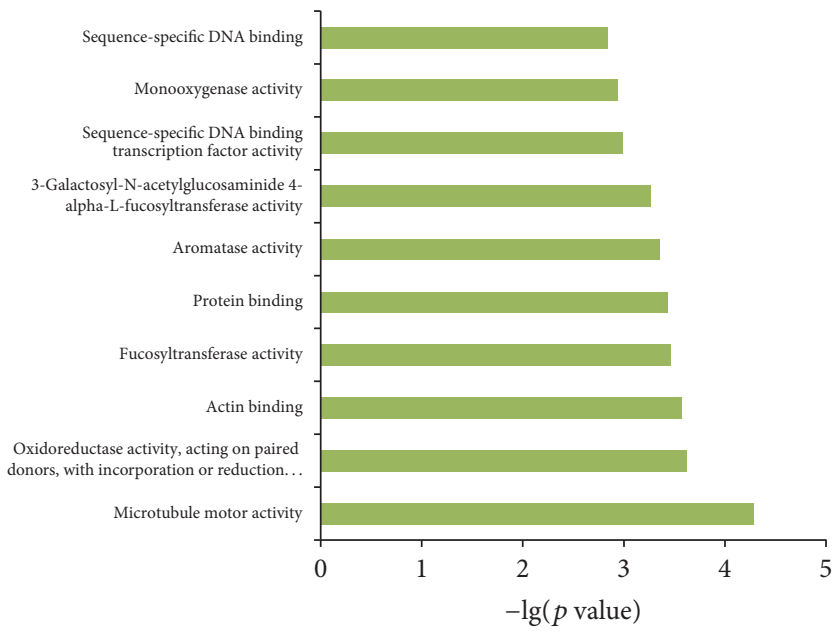

(c)

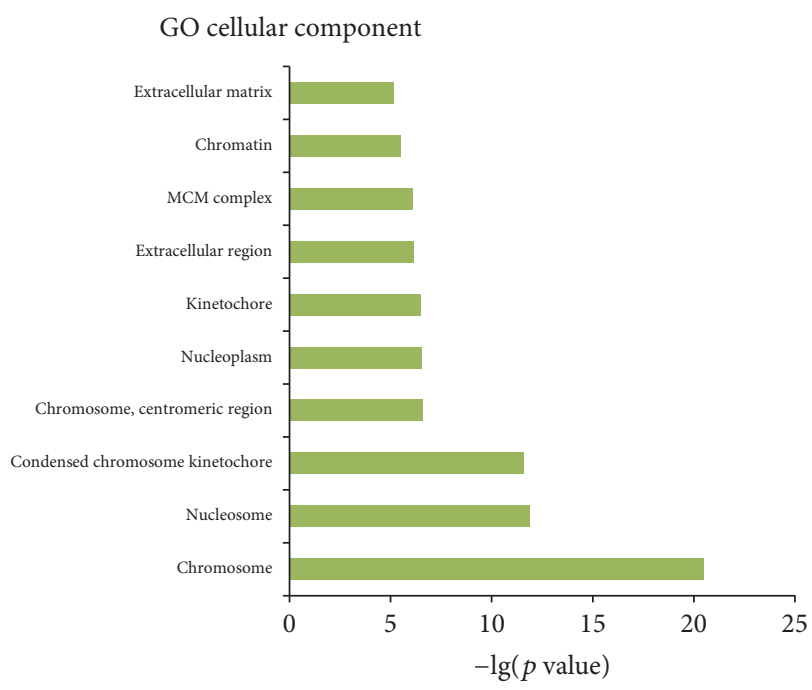

(b)

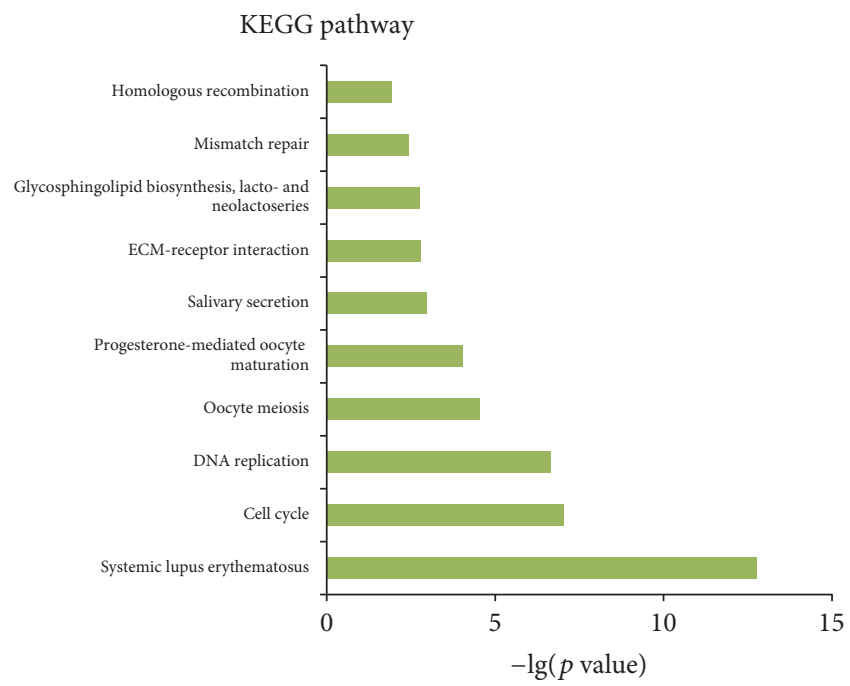

(d)

Figure 4: GO and KEGG pathway analysis. (a)-(c) Top 10 enrichment GO terms for differentially expressed mRNAs. The bar plot shows the enrichment scores ( $\lg (p$ value $)$ ) of the significant enrichment GO terms. (a) GO terms of biological process (BP); (b) GO terms of cellular component (CC); (c) GO terms of molecular function (MF). (d) Top 10 pathways of differentially expressed mRNAs in SSCC. The vertical axis represents the pathway category and the horizontal axis represents the enrichment score $(-\lg (p$ value $))$ of the pathway.

KEGG pathway analysis identified that many pathways were related to cancer, such as microRNAs in cancer, P53 signaling pathway, PI3K-Akt signaling pathway. For example, it has been documented that the p53 signaling pathway is activated in many solid tumors, including SSCC [1, 16, 17]. In the present study, p53 signaling pathway was related to IncRNA NONHSAT125629. This molecule may participate in numerous biological processes, including mitotic cell cycle, cell division, DNA replication, G1/S transition of mitotic cell cycle, and G2/M transition of mitotic cell cycle.

The global network (gene signal-network) and lncRNAmRNA network structure analysis were established to show the core genes that play a critical role in this SSCC gene network. However, how these genes participate in the pathogenesis of SSCC largely remains unknown. The analysis revealed that MAPK12, RAPGEF3, and KIT exhibited the most betweenness centrality and all were related to the cancer progression. Thus, our preliminary data provide a justification for the involvement of these genes in SSCC development. For example, KIT is associated with various pathways related to cancer, such as pathways in cancer and PI3K-Akt signaling pathway. Mutations in this gene are associated with gastrointestinal stromal tumors [18], lung cancer [19], and breast cancer [20].

The limitation of this study lies in the fact that the sample size is relatively small. Our results require further validation in larger prospective patient cohorts and functional experiments, both in vitro and in vivo. Our results provide some valuable clues for future function and mechanism studies of SSCC. 


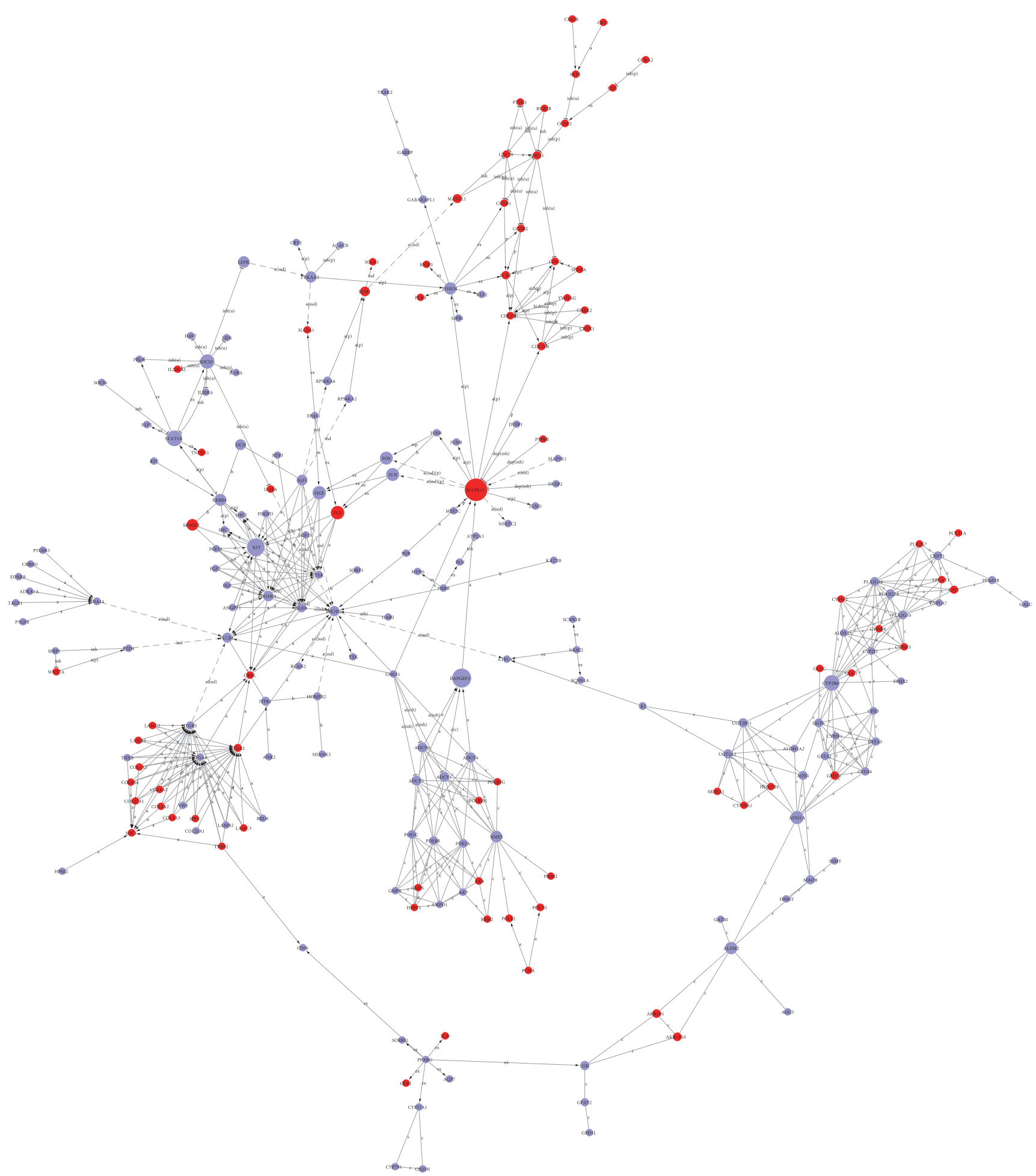

FIGURE 5: Signal-net. The interaction network of differentially expressed genes (signal-net). The circles represent important functional genes in SSCC (blue: downregulated genes; red: upregulated genes); the circle size represents the degree of interaction (betweenness centrality); the lines indicate the interactions.

\section{Conclusions}

In summary, to our knowledge, our study is the first screening and analysis of lncRNA expression profile in SSCC. The results show that genes regulated by these lncRNAs are involved in cancer pathways as a proof of principle. This may offer new insights into pathogenesis and could be a promising way to dissect the molecular pathogenesis of this refractory cancer. Our study lays the foundation for further investigation of this disease. Further large scale studies are 
TABLE 4: Top 20 aberrantly expressed mRNAs in microarray for 6 pairs of SSCC and paired adjacent noncancerous sinonasal tissues.

\begin{tabular}{|c|c|c|c|c|c|c|}
\hline Probe name & $p$ & $\mathrm{FC}(\mathrm{abs})$ & Regulation & Gene symbol & Chr & Genbank accession \\
\hline A_33_P3265783 & 0.015708 & 379.6405 & Down & STATH & chr4 & NM_003154 \\
\hline A_23_P154784 & 0.043979 & 159.44 & Down & BPIFB1 & $\operatorname{chr} 20$ & NM_033197 \\
\hline A_33_P3300312 & 0.034496 & 146.1192 & Down & DMBT1 & chr10 & NM_007329 \\
\hline A_24_P844984 & 0.030927 & 119.5701 & Down & PIGR & chrl & NM_002644 \\
\hline A_23_P86599 & 0.023373 & 95.27439 & Down & DMBT1 & $\operatorname{chr} 10$ & NM_007329 \\
\hline A_24_P146683 & 0.032317 & 89.55136 & Down & MSMB & chr10 & NM_002443 \\
\hline A_33_P3216570 & 0.003716 & 75.59971 & Down & MUC5AC & $\mathrm{N} / \mathrm{A}$ & AJ298317 \\
\hline A_23_P218369 & $3.92 E-04$ & 73.96607 & Down & CCL14 & $\operatorname{chr17}$ & NM_032963 \\
\hline A_23_P118203 & 0.017284 & 68.04929 & Down & ZG16B & chr16 & NM_145252 \\
\hline A_21_P0013344 & 0.032196 & 61.89528 & Down & AZGP1 & $\operatorname{chr} 7$ & NM_001185 \\
\hline A_23_P95930 & $6.01 E-05$ & 58.4917 & Up & HMGA2 & $\operatorname{chr} 12$ & NM_003483 \\
\hline A_32_P173662 & 0.002064 & 58.24841 & Down & CRISP2 & chr6 & NM_003296 \\
\hline A_23_P362694 & 0.036618 & 54.65685 & Down & C4orf7 & $\operatorname{chr} 4$ & NM_152997 \\
\hline A_33_P3245228 & 0.031988 & 52.29204 & Down & BPIFA1 & $\operatorname{chr} 20$ & NM_130852 \\
\hline A_23_P58228 & 0.017304 & 50.18366 & Down & ODAM & $\operatorname{chr} 4$ & NM_017855 \\
\hline A_23_P429998 & $1.13 E-04$ & 48.27517 & Down & FOSB & $\operatorname{chr} 19$ & NM_006732 \\
\hline A_33_P3233040 & 0.012252 & 42.21305 & Down & SERPINB11 & $\operatorname{chr} 18$ & NM_080475 \\
\hline A_23_P8702 & 0.018377 & 40.33374 & Down & PIP & chr7 & NM_002652 \\
\hline A_33_P3275702 & $1.49 E-05$ & 33.86336 & Down & FMO2 & chr1 & NM_001460 \\
\hline A_21_P0000003 & 0.006532 & 31.00913 & Down & PRR4 & chr12 & NM_007244 \\
\hline
\end{tabular}

FC, fold change; Chr, chromosome; NA, not annotated.

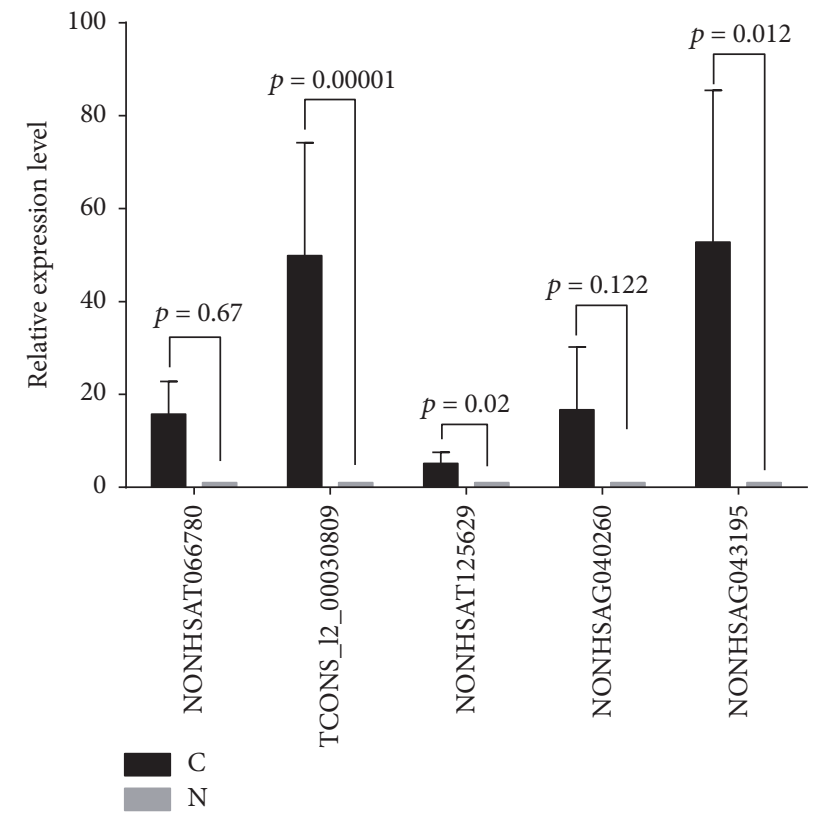

FIGURE 6: qRT-PCR validation. qRT-PCR verification of 5 candidate lncRNAs in 22 pairs of SSCC tissues. The $y$-axis represents the relative expression levels of lncRNAs. Paired $t$-tests (2-tailed) were performed to compare the expression levels between carcinoma (C) and noncancerous tissues $(\mathrm{N})$, and a $p$ value $<0.05$ indicated statistical significance.

warranted to provide convincing evidence for clarifying the functions of lncRNAs in SSCC and determining whether these lncRNAs can serve as new diagnostic biomarkers, prognostic factors for survival, and therapeutic targets in SSCC.

\author{
Abbreviations \\ lncRNA: Long noncoding RNA \\ SSCC: $\quad$ Sinonasal squamous cell carcinoma \\ qRT-PCR: Quantitative real-time polymerase chain \\ reaction \\ GO: $\quad$ Gene ontology \\ KEGG: Kyoto Encyclopedia of Genes and \\ Genomes \\ TNM: Tumor-node-metastasis \\ AJCC: American Joint Committee on Cancer \\ FC: $\quad$ Fold change \\ Chr: Chromosome \\ N/A: $\quad$ Not annotated.
}

\section{Disclosure}

The present address of Ju-gao Fang is Dongjiaominxiang 1st, Dongcheng District, Beijing 100730, China. The present address of Yong-xiang Wei is 2nd Anzhen Road, Chaoyang District, Beijing 100029, China.

\section{Competing Interests}

The authors declare that they have no conflict of interests. 


\section{Acknowledgments}

The authors gratefully acknowledge the assistance of Jayant M. Pinto, M.D., Associate Professor, Section of Otolaryngology-Head and Neck Surgery, Department of Surgery, The University of Chicago, who provided useful intellectual input. This work was supported by Beijing Municipal Administration of Hospitals Clinical Medicine Development Special Funding (Grant no. 201311).

\section{References}

[1] C. García-Inclán, A. López-Hernández, M. Alonso-Guervós et al., "Establishment and genetic characterization of six unique tumor cell lines as preclinical models for sinonasal squamous cell carcinoma," Scientific Reports, vol. 4, article 4925, 2014.

[2] J. H. Turner and D. D. Reh, "Incidence and survival in patients with sinonasal cancer: a historical analysis of population-based data," Head and Neck, vol. 34, no. 6, pp. 877-885, 2012.

[3] B. Ansa, M. Goodman, K. Ward et al., "Paranasal sinus squamous cell carcinoma incidence and survival based on surveillance, epidemiology, and end results data, 1973 to 2009," Cancer, vol. 119, no. 14, pp. 2602-2610, 2013.

[4] K. Zhu, R. S. Levine, E. A. Brann, H. I. Hall, L. S. Caplan, and D. R. Gnepp, "Case-control study evaluating the homogeneity and heterogeneity of risk factors between sinonasal and nasopharyngeal cancers," International Journal of Cancer, vol. 99, no. 1, pp. 119-123, 2002.

[5] L. Alos, S. Moyano, A. Nadal et al., "Human papillomaviruses are identified in a subgroup of sinonasal squamous cell carcinomas with favorable outcome," Cancer, vol. 115, no. 12, pp. 27012709, 2009.

[6] X. Lan, H. Zhang, Z. Wang et al., "Genome-wide analysis of long noncoding RNA expression profile in papillary thyroid carcinoma," Gene, vol. 569, no. 1, pp. 109-117, 2015.

[7] C. Chen, Z. Li, Y. Yang, T. Xiang, W. Song, and S. Liu, "Microarray expression profiling of dysregulated long noncoding RNAs in triple-negative breast cancer," Cancer Biology \& Therapy, vol. 16, no. 6, pp. 856-865, 2015.

[8] Q.-Q. Yang and Y.-F. Deng, "Genome-wide analysis of long non-coding RNA in primary nasopharyngeal carcinoma by microarray," Histopathology, vol. 66, no. 7, pp. 1022-1030, 2015.

[9] X. Shen, B. Xie, Z. Ma et al., "Identification of novel long noncoding RNAs in triple-negative breast cancer," Oncotarget, vol. 6, no. 25, pp. 21730-21739, 2015.

[10] N. Nohata, T. Hanazawa, N. Kikkawa et al., "Identification of novel molecular targets regulated by tumor suppressive miR-1/miR-133a in maxillary sinus squamous cell carcinoma," International Journal of Oncology, vol. 39, no. 5, pp. 1099-1107, 2011.

[11] N. Nohata, T. Hanazawa, N. Kikkawa et al., "Tumour suppressive microRNA-874 regulates novel cancer networks in maxillary sinus squamous cell carcinoma," British Journal of Cancer, vol. 105, no. 6, pp. 833-841, 2011.

[12] T. Kinoshita, N. Nohata, H. Yoshino et al., “Tumor suppressive microRNA-375 regulates lactate dehydrogenase B in maxillary sinus squamous cell carcinoma," International Journal of Oncology, vol. 40, no. 1, pp. 185-193, 2012.

[13] W. Gao, J. Y.-W. Chan, and T.-S. Wong, "Long non-coding RNA deregulation in tongue squamous cell carcinoma," BioMed Research International, vol. 2014, Article ID 405860, 2014.
[14] Z. Shen, Q. Li, H. Deng, D. Lu, H. Song, and J. Guo, "Long noncoding RNA profiling in laryngeal squamous cell carcinoma and its clinical significance: potential biomarkers for LSCC," PLoS ONE, vol. 9, no. 9, Article ID e108237, 2014.

[15] W. Zhang, L. Wang, F. Zheng et al., "Long noncoding RNA expression signatures of metastatic nasopharyngeal carcinoma and their prognostic value," BioMed Research International, vol. 2015, Article ID 618924, 13 pages, 2015.

[16] M.-B. Franzmann, C. Buchwald, G. K. Jacobsen, and H. Lindeberg, "Expression of p53 in normal nasal mucosa and in sinonasal papillomas with and without associated carcinoma and the relation to human papillomavirus (HPV)," Cancer Letters, vol. 128, no. 2, pp. 161-164, 1998.

[17] R. Holmila, J. Bornholdt, P. Heikkilä et al., "Mutations in TP53 tumor suppressor gene in wood dust-related sinonasal cancer," International Journal of Cancer, vol. 127, no. 3, pp. 578-588, 2010.

[18] S. Rossi, M. Sbaraglia, M. C. Dell'Orto et al., "Concomitant $\mathrm{KIT} / \mathrm{BRAF}$ and PDGFRA/BRAF mutations are rare events in gastrointestinal stromal tumors," Oncotarget, vol. 7, no. 21, pp. 30109-30118, 2016.

[19] L. Booth, J. L. Roberts, M. Tavallai et al., "The afatinib resistance of in vivo generated $\mathrm{H} 1975$ lung cancer cell clones is mediated by SRC/ERBB3/c-KIT/c-MET compensatory survival signaling," Oncotarget, vol. 7, no. 15, pp. 19620-19630, 2016.

[20] J. Shao, L. Gan, and J. Wang, “Transfection of chondromodulin I into human breast cancer cells and its effect on the inhibition of cancer cell growth," Molecular Medicine Reports, vol. 13, no. 5, pp. 4303-4308, 2016. 


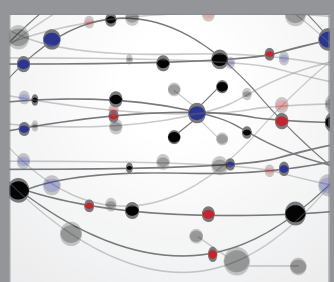

The Scientific World Journal
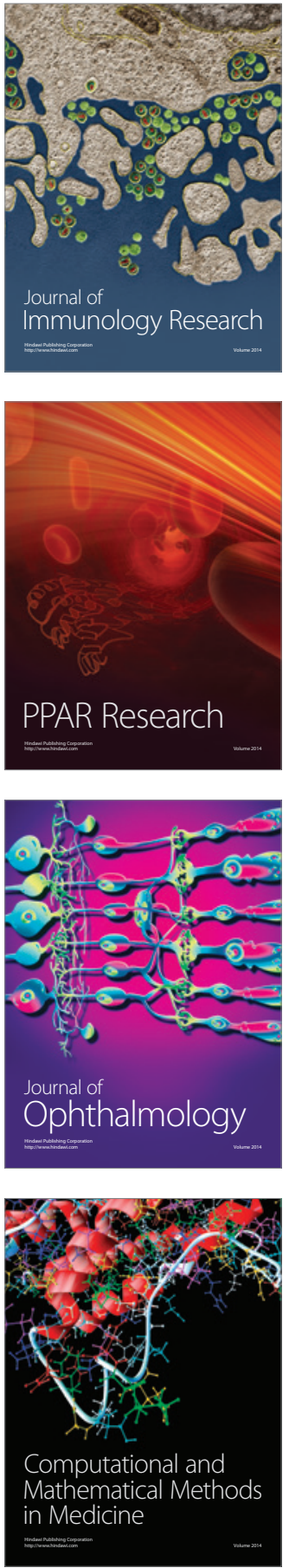

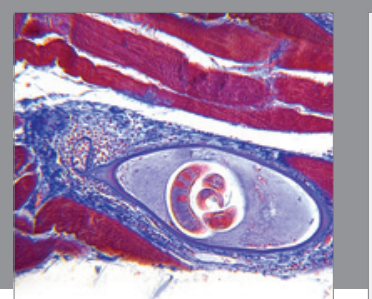

Gastroenterology Research and Practice

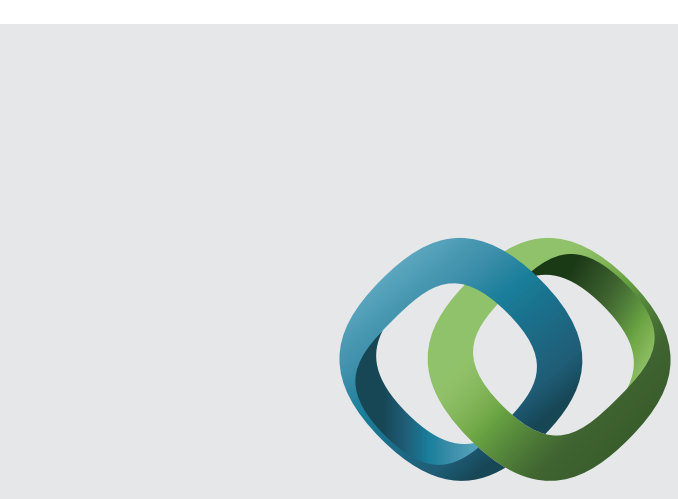

\section{Hindawi}

Submit your manuscripts at

http://www.hindawi.com
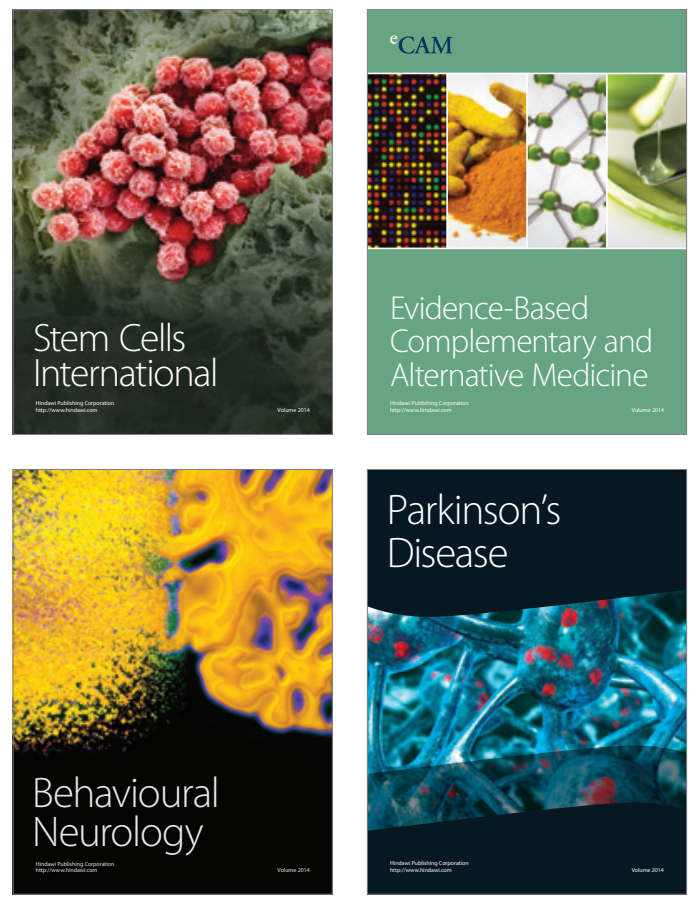
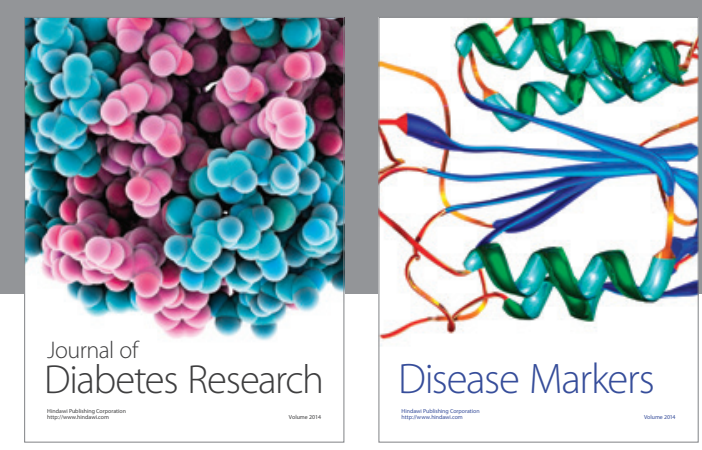

Disease Markers
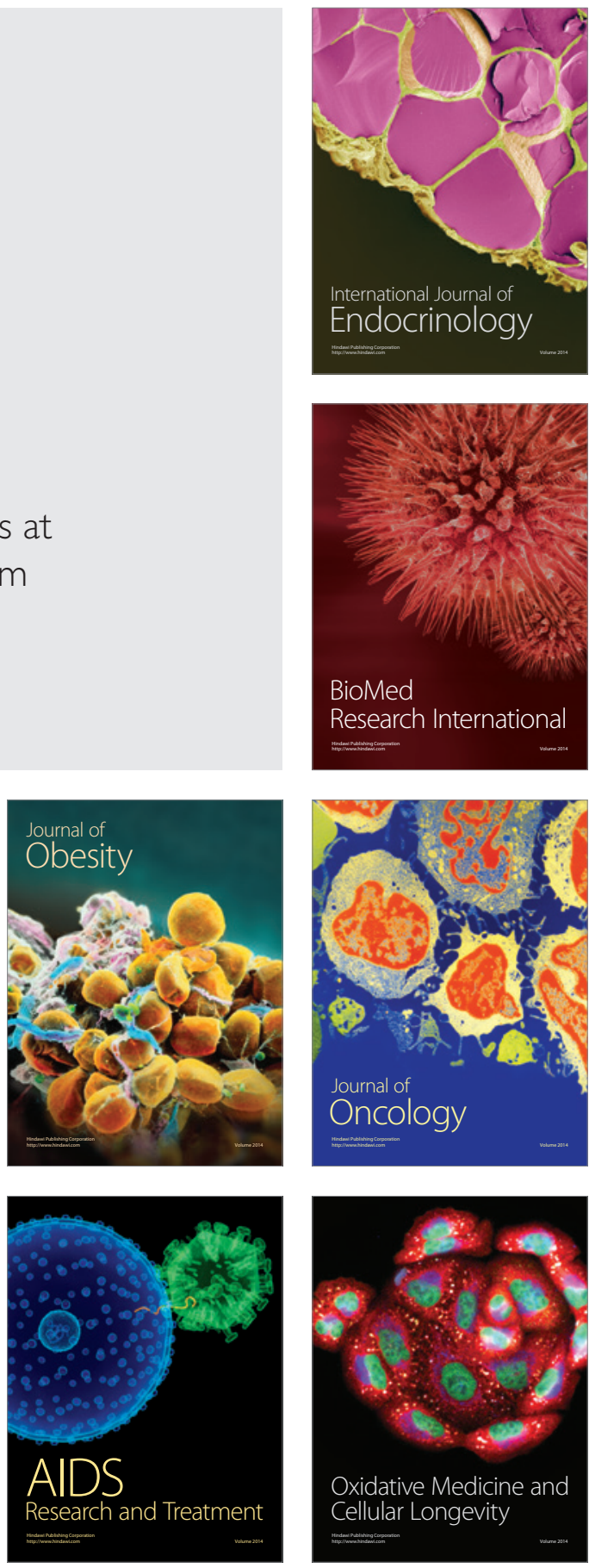